\title{
SCREENING AND MONITORING FOR BLADDER CANCER: REFINING THE USE OF NMP22
}

\author{
LEE E. PONSKY, SHASHIKALA SHARMA, LAKSHMI PANDRANGI, SUMITA KEDIA, \\ DAVID NELSON, ASHOK AGARWAL AND CRAIG D. ZIPPE*,$\dagger$ \\ From the Cleveland Clinic Urological Institute, Andrology-Oncology Research Laboratory and Biostatistics and Epidemiology, The \\ Cleveland Clinic Foundation, Cleveland, Ohio
}

\section{ABSTRACT}

Purpose: While detecting bladder cancer, bladder tumor markers demonstrate improved sensitivity compared with urinary cytology but the current limitation is the low specificity and positive predictive value, that is high false-positive rate. We examined the clinical categories of the false-positive results, established relative exclusion criteria, and recalculated the specificity and positive predictive value of this assay with these criteria.

Materials and Methods: A total of 608 patients considered at risk for bladder cancer presented to a urology clinic and submitted a single urine sample. Of the 608 patients 529 (87\%) presented with de novo hematuria or chronic voiding symptoms without a diagnosis of bladder cancer. There were 79 $(13.0 \%)$ patients being monitored with a known history of bladder cancer. Each urine sample was examined via cytology, urinalysis, culture and NMP22 $\div$ protein assay. All patients underwent office cystoscopy, and transurethral resection and/or biopsy if a bladder tumor was suspected.

Results: Of the 608 patients $226(37.2 \%)$ presented with microscopic hematuria, $143(23.5 \%)$ with gross hematuria and 239 (39.3\%) had chronic symptoms of urinary frequency or dysuria. There were $52(8.6 \%)$ patients who had histologically confirmed bladder cancer. Of these 52 cancers NMP22 detected 46 (88.5\%), whereas cytology identified only $16(30.8 \%)$. When atypical cytology was considered positive, cytology detected $32(61.5 \%)$ cases. In the 135 patients with increased NMP22 values the 46 identified tumors were accompanied by 89 false-positive values yielding a specificity of $83.9 \%$ and a positive predictive value of $34.1 \%$. These false-positive results were divided into 6 clinical categories. Exclusion of these categories improved the specificity and positive predictive value of NMP22 to $99.2 \%$ and $92.0 \%$, respectively, yielding results similar to urinary cytology $(99.8 \%$ and $94.1 \%)$.

Conclusions: Awareness and exclusion of the categories of false-positive results can increase the specificity and positive predictive value of NMP22, enhancing the clinical use of this urinary tumor marker.

KEY WoRDs: bladder neoplasms; tumor markers, biological; antigens, neoplasm; carcinoma, transitional cell; nuclear matrix

Early diagnosis of bladder cancer allows for effective local treatment and optimizes the success of surgical therapy. In the majority of patients successful treatment of superficial bladder cancer can be accomplished with minimally invasive procedures, such as transurethral resection or fulguration, precluding the need for more aggressive surgical therapy. Survival rate reflects the importance of early diagnosis. Detected at the superficial stage, the 5-year survival rate for patients with clinical stage $\mathrm{Ta}$ and $\mathrm{T} 1$ bladder cancer ranges from $82 \%$ to $95 \%$, while corresponding survival rates for those with muscle invasive or metastatic disease are $50 \%$ and $6 \%$, respectively. ${ }^{1-5}$

Many researchers have tried to evaluate noninvasive methods to accurately and easily identify the presence of bladder cancer. ${ }^{6-14} \mathrm{~A}$ number of diagnostic urinary tumor markers evolving from new molecular technologies are being

Accepted for publication January 12, 2001.

* Requests for reprints: Cleveland Clinic Urologic Institute, A-100, Cleveland Clinic Foundation, 9500 Euclid Ave., Cleveland, Ohio 44195.

$\dagger$ Financial interest and/or other relationship with Pfizer, AstraZeneca and TAP.

Editor's Note: This article is the second of 5 published in this issue for which category $1 \mathrm{CME}$ credits can be earned. Instructions for obtaining credits are given with the questions on pages 246 and 247. tested for screening specific high risk patients. In our study the efficacy of 1 tumor assay (NMP22) was evaluated as a screening modality in high risk patients as well as a monitoring modality in those with a known history of bladder cancer. Patients were considered to be at risk for bladder cancer based on the presence of microscopic or gross hematuria, or chronic irritative voiding symptoms such as frequency or dysuria.

Urinary cytology lacks sensitivity, while office cystoscopy lacks specificity. The sensitivity of voided urine cytology ranges from $40 \%$ to $50 \%$ in high grade disease but is reported to be as low as $20 \%$ to $30 \%$ in low grade low stage disease. ${ }^{15-17}$ Although urinary cytology has been the reference standard for noninvasive testing, the low sensitivity misses a significant number of cancers. This low sensitivity may result in a delay in diagnosis. On the other hand, while cystoscopy remains the reference standard for invasive testing, the primary indication for cystoscopy, which is hematuria, has a low specificity rendering it an inefficient tool for screening bladder cancer. ${ }^{15}$

The recent introduction of urinary tumor markers potentially challenges the efficacy of the current diagnostic evaluation. In a series of recent studies researchers evaluated the efficacy of urinary tumor markers for detection of recurrent bladder cancer. ${ }^{15-17}$ These studies show excellent sensitivity with these new urinary markers, particularly the ability to 
detect low grade low stage tumors. Sensitivity of these urinary tumor markers was reported to be 2 to 3 times greater than that of cytology, which translates into improved cancer detection. Despite excellent sensitivity, the limitations of these urinary markers have been low specificity and, more importantly, low positive predictive value. Specificity is frequently cited as an efficacy measure for screening tests.

We retrospectively attempt to clarify some of the confusion surrounding the high incidence of false-positive results that occurred with the NMP22 assay in patients at risk for bladder cancer. The NMP22 assay is a double monoclonal antibody immunoassay for the quantitative measurement of the nuclear mitotic apparatus protein designated NMP22, which is performed on stabilized urine samples. It has been approved by the Food and Drug Administration for detection of occult or recurrent transitional cell carcinoma after transurethral resection. We examine the clinical categories of falsepositive results contributing to the low positive predictive values, develop relative categorical exclusion criteria, and omit the patients identified by these criteria, recalculating the specificity and positive predictive value with these criteria.

\section{MATERIALS AND METHODS}

The institutional review board approved our study and patients provided written informed consent. Between May 1996 and December 1998 patients were evaluated at the urology clinic at 1 institution for unresolved symptoms of gross hematuria in 143, microscopic hematuria in 226 or chronic irritative voiding symptoms, such as frequency and dysuria, in 239 patients. Of the 608 patients 529 (87\%) presented with de novo hematuria or chronic voiding symptoms without a diagnosis of bladder cancer, and $79(13.0 \%)$ were being monitored with a known history of bladder cancer. Of the patient population $72 \%$ were males and $28 \%$ were females. Mean patient age (plus or minus standard deviation) was $70.4 \pm 10.6$ and $61.2 \pm 12.8$ years in the malignant and benign groups, respectively. Patients were queried to determine whether there was any known history of bladder cancer or genitourinary cancer, concurrent or recent urinary tract infection, history of urinary calculi, or the presence of any foreign body in urinary tract, that is stent and nephrostomy tube.

All 608 patients were evaluated by urinalysis, urine culture, voided urine cytology, the NMP22 test kit and office cystoscopy. If a potential malignancy was observed on office cystoscopy, the patient was scheduled while in the operating room for transurethral resection and/or biopsy. All reported bladder cancers were confirmed by histological evaluation. All cystoscopies and surgical procedures were performed by 1 urologist, and all urinary tests were performed at our institution. The cytopathologist was blinded to the NMP22 results. Each patient submitted a single urine sample before undergoing office cystoscopy. Each sample was divided into 2 aliquots, including 1 that was stabilized with the NMP22 test kit stabilizer and assayed for NMP22, and the other was sent for cytological examination, urinalysis and culture. ${ }^{15}$

An NMP22 value greater than 10 units per ml. was designated as positive for potential urothelial malignancy in our screened high risk patients who presented with hematuria or chronic irritative voiding symptoms. The cutoff value of 10 units per ml. was determined to be optimal by a receiver operator characteristics analysis performed in previous studies by our group. ${ }^{15,18}$ In the patients monitored with a history of bladder cancer a cutoff value of greater than 6.4 units per ml. was used, as suggested by previous investigators. ${ }^{15,16,19}$

Sensitivity and specificity were calculated before and after application of exclusion criteria. Comparisons of sensitivity and specificity were performed with McNemar's test and comparisons of predictive value rates were performed with Fisher's exact test. All calculations were performed with commercial software.

\section{RESULTS}

Of the 608 patients evaluated $52(8.6 \%)$ had histologically confirmed bladder cancer (table 1). Of the 52 tumors NMP22 detected $46(88.5 \%)$, the presence of microscopic and gross hematuria detected $42(82.7 \%)$ and urinary cytology identified only $16(30.8 \%)$. In the remaining 36 cancer cases urinary cytology was atypical in 16 and negative in 20 . If atypical cytology was treated as positive, cytology then detected $32(61.5 \%)$ tumors. Of the patients with histologically confirmed cancer 40 had a history of bladder cancer.

In the 135 patients with increased NMP22 values 46 tumors were detected with 89 false-positive values, yielding a specificity of $83.9 \%$ and a positive predictive value of $34.1 \%$ (table 2 ). In contrast, urinary cytology alone had a specificity and positive predictive value of $99.8 \%$ and $94.1 \%$, respectively. When atypical cytology was treated as positive, the sensitivity increased from $30.7 \%$ to $61.5 \%(\mathrm{p}<0.01)$ but the specificity and positive predictive value dropped to $84.7 \%$ and $61.9 \%$, respectively ( $\mathrm{p}<0.01$, table 3 ).

The 52 tumors were predominantly superficial in stage and grade, including 30 that were stage Ta and grade 1 to 2,3 were pure carcinoma in situ, 12 were stage T1 and grade 2 to 3 , and 7 were stage T2 and grade 3 or greater. The median NMP22 value in the malignancy group was 22.8 units per ml. (4.3 to 186.6). The 6 tumors that NMP22 failed to detect were all superficial in nature, including 4 solitary stage $\mathrm{Ta}, 1$ multifocal stage Ta and 1 stage T1 tumor. In contrast, 8 of the $36(22.2 \%)$ tumors not diagnosed by cytology were stage T1 or greater. There was 1 unexplained false-positive cytology that failed to show any malignancy after random biopsies.

We observed that the false-positive results could be grouped into 6 clinical categories (table 4). A false-positive rate for each of these clinical categories was calculated. These 6 categories and respective false-positive rates were benign inflammatory or infectious conditions, including cystitis, recurrence, urinary tract infection and prostatitis, in $43.8 \%$, the presence of renal or bladder calculi in $83.3 \%$, current or recent history of a foreign body in the urinary tract, including stent and nephrostomy tube, in $100 \%$, any bowel interposition segment, including ileal conduit and continent diversion, in $100 \%$, other genitourinary cancer, including prostate and renal cell, in $20.7 \%$, and an instrumented urinary sample in $26.5 \%$ of cases. A seventh category of "no known pathology" was included as a control and had a falsepositive rate of $0.85 \%$. Mean NMP22 values in the 6 exclusion categories were all at least 2 to 3 times higher than the cutoff values for positive results. The first 4 exclusion categories, including benign inflammatory conditions, renal and bladder calculi, and presence of foreign bodies and bowel segments, exhibited false-positive rates ranging from $44 \%$ to $100 \%$ and should be considered absolute exclusion criteria. The remaining 2 categories, including other genitourinary cancer and instrumentation, exhibited false-positive rates of $20.7 \%$ and $26.5 \%$, respectively, and should be considered as relative exclusion criteria.

TABLE 1. Overall results of cystoscopy, NMP22 assay and voided uring cytology

\begin{tabular}{|c|c|c|c|c|c|}
\hline & \multirow{2}{*}{ No. Pts. } & \multirow{2}{*}{$\begin{array}{l}\text { No. Abnormal } \\
\text { NMP22 } \\
\text { Results }\end{array}$} & \multicolumn{3}{|c|}{ No. Cytologies } \\
\hline & & & Pos. & Neg. & Atypical \\
\hline Benign & 556 & 89 & 1 & 471 & 84 \\
\hline Malignant & 52 & 46 & 16 & 20 & 16 \\
\hline Totals & 608 & 135 & 17 & 491 & 100 \\
\hline
\end{tabular}


TABLE 2. Comparison of NMP22 assay results before and after exclusion of false-positive categories versus voided urinary cytology

\begin{tabular}{lccc}
\hline & \multicolumn{2}{c}{$\%$ NMP22 Results } & \\
\cline { 2 - 3 } & Before & After & \% Cytology \\
\hline Sensitivity & 88.5 & 88.5 & $30.7^{*}$ \\
Specificity & 83.9 & $99.2 \dagger$ & 99.8 \\
Pos. predictive value & 34.1 & $92.0 \ddagger$ & 94.1 \\
Neg. predictive value & 98.7 & 98.8 & 93.9 \\
\hline
\end{tabular}

* Versus NMP22 values $\mathrm{p}<0.01$.

$\dagger$ Versus berfore exclusion criteria $\mathrm{p}<0.01$

$\ddagger$ Versus before exclusion criteria $\mathrm{p}<0.05$.

TABLE 3. Analysis of positive cytology alone versus atypical and positive cytology combined

\begin{tabular}{lcc}
\hline & \% Pos. Cytology & \% Atypical Pos. Cytology \\
\hline Sensitivity & 30.7 & $61.5^{*}$ \\
Specificity & 99.8 & 84.7 \\
Pos. predictive value & 94.1 & $64.9^{*}$ \\
Neg. predictive value & 93.9 & 85.6 \\
\hline
\end{tabular}

*Versus positive cytology alone $\mathrm{p}<0.01$.

TABLE 4. Mean NMP22 values and false-positive rates for 6 clinical exclusion categories

\begin{tabular}{lcrrc}
\hline & $\begin{array}{c}\text { Mean NMP22 } \\
\text { (units/ml. }\end{array}$ & $\begin{array}{c}\text { No. } \\
\text { Folse- } \\
\text { Pos. }\end{array}$ & $\begin{array}{c}\text { No. True \% } \\
\text { Neg. }\end{array}$ & $\begin{array}{c}\text { False-Pos. } \\
\text { Rate* }\end{array}$ \\
\hline Benign inflammatory conditions & 28.6 & 28 & 64 & 43.8 \\
Renal bladder calculi & 65.4 & 10 & 2 & 83.3 \\
Presence or history foreign body & 148.4 & 8 & 0 & 100 \\
Bowel interposition segment & 162.8 & 14 & 0 & 100 \\
Other genitourinary Ca & 32.4 & 12 & 58 & 20.7 \\
Instrumentation & 26.2 & 13 & 36 & 26.5 \\
No known clinical pathology & 14.4 & 4 & 467 & 0.85 \\
\hline
\end{tabular}

*False-positive/false-positive plus true negative.

When patients from these 6 categories were excluded from the data analysis, the sensitivity remained the same as expected at $88.5 \%$ but the specificity improved from $83.9 \%$ to $99.2 \%(\mathrm{p}<0.05)$, and the positive predictive value improved from $34.1 \%$ to $92.0 \%$ ( $p<0.01$ ). The negative predictive value remained constant at $90.8 \%$, indicating that no additional tumors were missed by the application of these exclusion criteria.

\section{DISCUSSION}

We retrospectively examined the cancer detection rate and false-positive results generated by the NMP22 tumor marker assays while screening patients at risk for bladder cancer. We determined that by excluding patients with associated clinical problems from analysis, the specificity and positive predictive value of the NMP22 assay could be increased to $99.2 \%$ and $92.0 \%$ ( $\mathrm{p}<0.05$ and $<0.01$ ), respectively, values comparable to that of voided urine cytology (table 2). By excluding clinical categories of false-positive results, the specificity and positive predictive value of the NMP22 test were significantly improved while maintaining excellent sensitivity. With the application of these exclusion criteria, the NMP22 assay approximates more closely the reference standard of an "ideal tumor marker," which is a test that yields a sensitivity and specificity of $90 \%$.

This refinement for the clinical application of this assay has significance for the early detection of patients with predisposing factors for bladder cancer. This test could potentially be used to screen patients having familial, occupational or behavioral risk factors. A high risk population could be defined as all males and females age 50 years or older who have chronic urinary frequency, occupational exposure to carcinogenic chemicals and, more importantly, a history of chronic cigarette use. Defining the proper high risk popula- tion could make this test an effective, noninvasive, low cost screening modality.

Analysis of the patients with false-positive results revealed that 6 associated conditions could be identified. These categories included benign inflammatory or infectious conditions, presence of renal or bladder calculi, current or recent history of foreign body in the urinary tract, any bowel interposition segment, other genitourinary cancer and an instrumented urinary sample. If these categories were applied as exclusion criteria, then the positive predictive value of the NMP22 assay increased from $34.1 \%$ to $92.0 \%$ and specificity increased from $83.9 \%$ to $99.2 \%$. In the clinical treatment of patients at risk for bladder cancer the positive predictive value is considered the most important statistic because it inversely correlates with the number of false-positive results and unnecessary cystoscopies. Most patients excluded by these criteria had exceptionally high NMP22 urine levels, with mean values between 26.2 and 162.8 units per ml., and false-positive rates between $20.7 \%$ and $100 \%$. Application of these exclusion criteria significantly improved the overall positive predictive value and specificity of the NMP22 assay.

The biological factors that contribute to falsely increased NMP22 values involve any process that produces rapid turnover of any epithelial cell. The NMP22 protein is a nonspecific nuclear matrix protein that is shed with any disease process that causes rapid cell turnover. This fact is supported by the clinical exclusion categories identified in our study. For example, any bowel interposition segment induces a rapid turnover or shedding of the epithelial cells, just as foreign bodies cause an inflammatory urothelial response and accounts for exfoliated cells. Inflammatory conditions, such as cystitis and prostatitis, also cause rapid epithelial cell turnover. Finally, instrumentation can cause trauma to the urothelial epithelial cells and produce an increased NMP22 value. The addition of atypical cytology as positive significantly improved the sensitivity of cytology $(30.7 \%$ to $61.5 \%$ ). Although this improved sensitivity is 2 -fold better than cytology alone, sensitivity remains considerably lower than that of the NMP22 test. Also, to achieve this improved sensitivity, the specificity and positive predictive value decreased to $84.7 \%$ and $64.9 \%$, respectively (table 3 ), which were significantly lower than the NMP22 assay values after exclusion criteria. For urologists who do not have access to the NMP22 test, treating atypical cytology as positive can significantly improve the cancer detection rate but at the cost of additional false-positive results, as reflected by the decrease in the positive predictive value from $94.1 \%$ to $64.9 \%$.

In a previous study at our laboratory the NMP22 assay was reported to have $100 \%$ sensitivity for early detection of bladder cancer. Such perfect accuracy is the exception rather than the rule. Other researchers report sensitivities ranging from $48 \%$ to $90.9 \% .^{10,15,17-20}$ Because sensitivity of the NMP22 marker typically is not $100 \%$, total elimination of diagnostic or surveillance cystoscopy cannot be advised. However, there is no question that reducing the number of invasive cystoscopies or lengthening the interval of them has use in the current environment of cost containment.

The availability of these urinary tumor markers will force us to reevaluate the current algorithm for the diagnostic evaluation of microhematuria and interval of surveillance cystoscopy. At a cost of less than $\$ 20$ in the United States, this assay is comparable to urinalysis and is significantly less than urinary cytology. With sensitivity 2 to 3 times that of cytology with a negative predictive value greater than $90 \%$, with the use of the exclusion criteria, this biomarker rarely misses a bladder malignancy. Although similar sensitivity $(82.7 \%)$ for cancer detection can be obtained for evaluating microscopic or gross hematuria, an important difference exists. The use of a urinary tumor marker with appropriate exclusion criteria can potentially render it cancer specific. Also, to our knowledge effective exclusion criteria for micro- 
scopic hematuria have not been developed. Therefore, the low positive predictive value or high false-positive rate must necessarily be accepted.

\section{CONCLUSIONS}

Exclusion of the categories of false-positive results can increase the specificity and positive predictive value of NMP22, enhancing clinical use of this urinary tumor marker. The current situation of NMP22 may be analogous to understanding the application and evolution of the prostate specific antigen (PSA) test to screen for prostate cancer. ${ }^{21}$ The serum PSA test was associated with a high false-positive rate or low positive predictive value. However, the low positive predictive value of PSA and, therefore, clinical use, improved with time as clinicians identified the pathological conditions, such as acute and chronic prostatitis and benign prostatic hyperplasia, which produced false-positive values. However, most urologists would agree that screening patients with the PSA test, despite the shortcomings, is the single most important advance in prostate cancer detection during the last decade. Similar to the evolution and significance of PSA testing, it is likely that the clinical use of urinary tumor markers will be further enhanced as definitive exclusion criteria are recognized. Moreover, biotechnological research will continue to look for a more specific cancer marker since urine testing is noninvasive, easily obtained and cost effective with current automation.

\section{REFERENCES}

1. Landis, S. H., Murray, T., Bolden, S. et al: Cancer statistics, 1999. CA Cancer J Clin, 49: 8, 1999

2. Cheng, L., Neumann, R. M., Weaver, A. L. et al: Predicting cancer progression in patients with stage $\mathrm{T} 1$ bladder carcinoma. J Clin Oncol, 17: 3182, 1999

3. Sanchez de la Muela, P., Rosell, D., Aguera, L. et al: Multivariate analysis of progression in superficial bladder cancer. $\mathrm{Br} \mathrm{J}$ Urol, 71: 284, 1993

4. Amling, C. L., Thrasher, J. B., Frazier, H. A. et al: Radical cystectomy for stages $\mathrm{Ta}$, Tis and $\mathrm{T} 1$ transitional cell carcinoma of the bladder. J Urol, 151: 31, 1994

5. Suzuki, K., Obata, K., Fukastsu, H. et al: Survival rate of bladder tumors: an analysis of 2304 patients with bladder tumors in the Tokai Urological Cancer Registry. Nippon Hinyokika Gakkai Zasshi, 81: 96, 1990

6. Steiner, G., Schoenberg, M. P., Linn, J. F. et al: Detection of bladder cancer recurrence by microsatellite analysis of urine.
Nat Med, 3: 621, 1997

7. Mao, L., Schoenberg, M. P., Scicchitano, M. et al: Molecular detection of primary bladder cancer by microsatellite analysis. Science, 271: 659, 1996

8. Ramakumar, S., Bhuiyan, J., Besse, J. A. et al: Comparison of screening methods in the detection of bladder cancer. J Urol, 161: 388,1999

9. Mian, C., Pycha, A., Wiener, H. et al: Immunocyt: a new tool for detecting transitional cell cancer of the urinary tract. J Urol, 161: 1486, 1999

10. Wiener, H. G., Mian, C. H., Haitel, A. et al: Can urine bound diagnostic tests replace cystoscopy in the management of bladder cancer? J Urol, 159: 1876, 1998

11. Getzenberg, R. H., Konety, B. R., Oeler, T. A. et al: Bladder cancer-associated nuclear matrix proteins. Cancer Res, 56: 1690,1996

12. Sarosdy, M. F., Hudson, M. A., Ellis, W. J. et al: Improved detection of recurrent bladder cancer using the Bard BTA stat test. Urology, 50: 349,1997

13. Soloway, M. S.: Editorial: do we have a prostate specific antigen for bladder cancer? J Urol, 161: 447, 1999

14. Akaza, H., Miyanaga, N., Tsukamoto, T. et al: Evaluation of urinary NMP22 (nuclear matrix protein 22) as a diagnostic marker for urothelial cancer-screening for urothelial cancer in patients with microscopic hematuria. Jap J Cancer Chemother, 24: 837, 1997

15. Sharma, S., Zippe, C. D., Pandrangi, L. et al: Exclusion criteria enhance the specificity and positive predictive value of NMP22 and BTA stat. J Urol, 162: 53, 1999

16. Landman, J., Chang, Y., Kavaler, E. et al: Sensitivity and specificity of NMP22, telomerase, and BTA in the detection of human bladder cancer. Urology, 52: 398, 1998

17. Miyanaga, N., Akaza, H., Tsukamoto, T. et al: Urinary nuclear matrix protein 22 (NMP22) as a marker for screening urothelial cancer in patients with microscopic hematuria. J Urol, suppl., 159: 243, abstract 931, 1998

18. Zippe, C., Pandrangi, L. and Agarwal, A.: NMP22 is a sensitive, cost-effective test in patients at risk for bladder cancer. J Urol, 161: 62,1999

19. Stampfer, D. S., Carpinito, G. A., Rodriguez-Villaneuva, J. et al: Evaluation of NMP22 in the detection of transitional cell carcinoma of the bladder. J Urol, 159: 394, 1998

20. Carpinito, G. A., Rukstalis, D. B., Pandrangi, L. V. et al: Prospective, multi-center study of NMP22 and cytology in patients with hematuria. J Urol, suppl., 159: 245 , abstract 937, 1998

21. Soloway, M. S., Briggman, V., Carpinito, G. A. et al: Use of a new tumor marker, urinary NMP22 in the detection of occult or rapidly recurring transitional cell carcinoma of the urinary tract following surgical treatment. J Urol, 156: 363, 1996 